\title{
Analytic models and forward scattering from accelerator to cosmic-ray energies
}

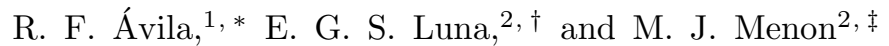 \\ ${ }^{1}$ Instituto de Matemática, Estatística e Computação Científica \\ Universidade Estadual de Campinas, 13083-970 Campinas, SP, Brazil \\ ${ }^{2}$ Instituto de Física Gleb Wataghin, \\ Universidade Estadual de Campinas, 13083-970 Campinas, SP, Brazil
}

(Dated: November 20, 2018)

\begin{abstract}
Analytic models for hadron-hadron scattering are characterized by simple analytical parametrizations for the forward amplitudes and the use of dispersion relation techniques to study the total cross section $\sigma_{\text {tot }}$ and the $\rho$ parameter (the ratio between the real and imaginary parts of the forward amplitude). In this paper we investigate simultaneously four aspects related to the application of the model to $p p$ and $\bar{p} p$ scattering, from accelerator to cosmic-ray energies: (1) the effect of different estimations for $\sigma_{t o t}$ from cosmic-ray experiments; (2) the differences between individual and global (simultaneous) fits to $\sigma_{\text {tot }}$ and $\rho ;(3)$ the role of the subtraction constant in the dispersion relations; (4) the effect of distinct asymptotic inputs from different analytic models. This is done by using as a framework the single Pomeron and the maximal Odderon parametrizations for the total cross section. Our main conclusions are the following: (1) Despite the small influence from different cosmic-ray estimations, the results allow us to extract an upper bound for the soft Pomeron intercept: $1+\epsilon=1.094$; (2) although global fits present good statistical results, in general, this procedure constraints the rise of $\sigma_{t o t}$; (3) the subtraction constant as a free parameter affects the fit results at both low and high energies; (4) independently of the cosmic-ray information used and the subtraction constant, global fits with the Odderon parametrization predict that, above $\sqrt{s} \approx 70$ $\mathrm{GeV}, \rho_{p p}(s)$ becomes greater than $\rho_{\bar{p} p}(s)$, and this result is in complete agreement with all the data presently available. In particular, we infer $\rho_{p p}=0.134 \pm 0.005$ at $\sqrt{s}=200 \mathrm{GeV}$ and $0.151 \pm 0.007$ at $500 \mathrm{GeV}$ (BNL RHIC energies). A detailed discussion of the procedures used and all the results obtained is also presented.
\end{abstract}

PACS numbers: 13.85.Dz, 13.85.Lg, 13.85.-t

\section{INTRODUCTION}

High-energy soft processes are presently a topical problem in high-energy physics, mainly because they are essentially a nonperturbative phenomenon [1]. Despite important results at the interface between soft and hard physics and recent progress in nonperturbative QCD, elastic scattering, the simplest soft process, cannot be described in a pure QCD framework, but only in a phenomenological context.

In this area, a variety of models, such as, for example, Regge, Diffraction, QCD-inspired models, and others [2], has survived due to a solid theoretical basis and efficiency in describing physical quantities. What is presently well established and accepted concerns some general principles, limits, bounds, and theorems, earlier deduced from the Mandelstam representation, potential scattering, and also from axiomatic field theories [3, 4]. In this context, analyticity, unitarity and crossing play central roles and are also the framework of several models referred to above. Among them, the so called analytic models are characterized by parametrizations of the hadronic amplitude through general analytic functions that strictly obey the formal principles and theorems. Specifically, the aim is to investigate two fundamental physical quantities that characterize the forward elastic scattering at high energies, namely, the total cross section $\sigma_{\text {tot }}$ and the $\rho$ parameter (the ratio of the real to the imaginary part of the forward elastic scattering amplitude). In terms of the scattering amplitude $F$ they may be given by

$$
\sigma_{t o t}(s)=\frac{\operatorname{Im} F(s, t=0)}{s}, \quad \rho(s)=\frac{\operatorname{Re} F(s, t=0)}{\operatorname{Im} F(s, t=0)},
$$

where $t$ is the four-momentum transfer squared and $s$ the center-of-mass energy squared. These expressions, in terms

\footnotetext{
*Electronic address: rfa@ifi.unicamp.br

$\dagger^{\dagger}$ Electronic address: luna@ifi.unicamp.br

${ }^{\ddagger}$ Electronic address: menon@ifi.unicamp.br
} 
of real and imaginary parts of the amplitude, obviously suggest dispersion relations as a suitable formal framework for investigations. For particle-particle and particle-antiparticle interactions, the addition of crossing symmetry extends and completes the analytical approach. It is expected that such a general formalism, avoiding details of the interaction or dynamics, could be a suitable tool in the search for adequate calculational schemes in nonperturbative QCD.

The analytic models have a long history and important results have been obtained through both integral relations [5], for example 6, 6, [8, 9], and derivative (analyticity) relations [10, 11, 12], for example [12, 13, 14]. Recently, much effort has been concentrated in the COMPETE Collaboration (COmputerized Models, Parameter Evaluation for Theory and Experiment), which joined the COMPAS group and others specialists also with outstanding contributions in the area. These authors have investigated a large class of analytic models, through several statistical indicators that complement the usual $\chi^{2}$ and and C.L. criteria. One of the main results is the universality of the $B \ln ^{2} s / s_{0}$ increase of the total cross section for all the collisions considered [14, 15].

Despite all the experience accumulated and the detailed analyses that have been developed, we understand that three aspects yet need some investigation and this is the main purpose of this work. These aspects are based on the following observations.

1. Beyond the energy region of the accelerators, experimental information on $p p$ total cross sections exists from cosmic-ray experiments. Despite the model dependence involved, the large error bars in the numerical results, and also the existence of discrepant values, some of these results are usually displayed as a support for several model predictions. However, this set of experimental information is not usually taken into account in explicitly quantitative analyses that could provide, for example, bounds for the increase of $\sigma_{\text {tot }}$ and $\rho$ (and, consequently, the intercept of exchanged trajectories) or for the differences between $p p$ and $\bar{p} p$ total cross sections (for exceptions, see [16, 17]). Moreover, as will be discussed in some detail, several results obtained by different authors, through different approaches, indicate nearly the same increase of $\sigma_{t o t}(s)$ and this increase is faster than usually believed or accepted.

2. In general, the fits are performed with the full hadronic amplitude, that is, simultaneous fits to $\sigma_{t o t}$ and $\rho$. Although some authors correctly claim that this procedure maximizes the number of data points, it must first be recalled that important results have been obtained through fits to only the $\sigma_{\text {tot }}$ data; a classical example is the approach by Donnachie and Landshoff [18. Moreover, as will be discussed, in the bulk of experimental data available, $\sigma_{\text {tot }}$ and $\rho$ have different status as physical quantities, since $\rho$ is estimated either by extrapolations through dispersion relations (and fits to $\sigma_{\text {tot }}$ data), or as a fit parameter to the differential cross section in the region of Coulomb-nuclear interference, and this is a delicate problem. Finally, since from the previous observation we shall also be interested in the high-energy cosmic-ray results, which concern only $\sigma_{\text {tot }}$ (and not $\rho$ ), global fits may constrain the possible increase of this quantity.

3. The connection between $\sigma_{\text {tot }}$ and $\rho$ through standard dispersion relations demands one subtraction [5]. Although the subtraction constant works as a fit parameter in traditional analysis [․ 7, [8, [9], it does not appear in the approach by the COMPETE Collaboration, since the derivative relation or prescription used does not involve subtraction constant. Once the analytical approach demands fits to experimental data and the free parameters involved are all correlated, it is expected that the presence or not of the subtraction constant may lead to different results.

The aim of this work is to investigate the above three observations and their inter-connections in a quantitative way. To this end, we shall analyze only $p p$ and $\bar{p} p$ elastic scattering, since for particle and antiparticle interactions they correspond to the highest energy interval with available data and are the only set including the cosmic-ray information on total cross sections ( $p p$ scattering). We shall use as framework two well known analytic models characterized by distinct asymptotic inputs, the Pomeron-Reggeon model by Donnachie and Landshoff [18] and the maximal Odderon model in the form discussed by Kang and Nicolescu [12. For the connections between real and imaginary parts of the forward amplitude we shall use derivative (analyticity) relations with one subtraction. Observation 1 is treated by the selection of two different ensembles of data from cosmic-ray results, observation 2 through individual and simultaneous fits to $\sigma_{t o t}$ and $\rho$, and observation 3 by treating the subtraction constant as a free fit parameter or assuming that its value is zero.

The manuscript is organized as follows. In Sec. II we present the experimental data to be analyzed and the criteria for the selection of two ensembles of data. In Sec. III we review the main formulas in the analytical approach, including the dispersion relations, the models to be used and some high-energy theorems. The fits and results for all the cases considered are presented in Sec. IV and the conclusions and final remarks in Sec. V. 


\section{EXPERIMENTAL INFORMATION AND ENSEMBLES}

In this section we review the experimental information that constitutes our data base (accelerator and cosmic-ray regions), together with discussions concerning the differences in the determination of $\sigma_{\text {tot }}$ and $\rho$, as well as the model dependences and discrepancies related to all the cosmic-ray information presently available. The criteria for selecting two ensembles of data are also presented and justified in detail.

\section{A. Accelerator data}

In the case of accelerator experiments, data on $\sigma_{\text {tot }}$ from $p p$ and $\bar{p} p$ scattering and extracted values for the $\rho$ parameter have been accumulated for a long time. Presently, experimental information extends up to $62.5 \mathrm{GeV}$ and $1.8 \mathrm{TeV}$ for $p p$ and $\bar{p} p$ scattering, respectively. The database, analyzed and compiled by the Particle Data Group (PDG), has become a standard reference and the corresponding readable files are available [19]. Since recent analysis has shown that general fits to these data are stable for $\sqrt{s}$ above $\sim 9 \mathrm{GeV}[14]$, we shall use here the sets for energies above $10 \mathrm{GeV}$; in our analysis the statistic and systematic errors are added linearly.

At this point, we briefly recall some differences in the determination of $\sigma_{\text {tot }}$ and $\rho[2]$, which suggest that they do not have the same status as physical quantities; this, in turn, corroborates our motivation to investigate global and individual fits separately.

First, one of the methods that was used to extract $\sigma_{\text {tot }}$ at the ISR did not depend on the $\rho$ value, but only on the machine luminosity and the rates of elastic and inelastic interactions. Therefore in these determinations $\sigma_{t o t}$ and $\rho$ are independent quantities. Other methods demand the determination of $\rho$ and the elastic scattering rate at small momentum transfer, extrapolated to the forward direction (slope parameter). In these cases, the quantities to be determined correspond to $\sigma_{\text {tot }}^{2}\left(1+\rho^{2}\right)$ (luminosity dependent) or $\sigma_{t o t}\left(1+\rho^{2}\right)$ (luminosity independent) [2]. In both cases, since it is known that $\rho<0.14, \sigma_{\text {tot }}$ may be obtained with reasonable accuracy even with a rough estimation of the $\rho$ parameter. Specifically, some authors use the $\rho$ value extrapolated from fits to $\sigma_{\text {tot }}$ and dispersion relations; in the other procedure, $\rho$ is determined from fits to the differential cross section data in the region of Coulomb-nuclear interference. In this case the determination is model dependent and it is interesting to note that the procedure, in the last instance, demands knowledge of how the hadronic exchanged object interacts, which is exactly what is looked for. This point seems clear in the Donnachie-Landshoff approach, since the authors do not use the $\rho$ data as input. We understand that all these facts reinforce the differences in the determination of $\rho$ and $\sigma_{\text {tot }}$, putting some limits on the interpretation of $\sigma_{t o t}$ and $\rho$ as physical quantities with the same status. Beyond these motivations for discriminating between individual and global fits, we add our interest in investigating cosmic-ray information, which concerns only $\sigma_{\text {tot }}$ and not $\rho$.

\section{B. Cosmic ray information}

For $p p$ collisions, the total cross section may also be inferred from cosmic-ray experiments and estimations exist in the high-energy interval $\sqrt{s}=6-40 \mathrm{TeV}$. The procedure is model dependent and different analyses lead to different results, as briefly reviewed in what follows.

The extraction of the proton-proton total cross section is based on the determination of the proton-air production cross section from analysis of extensive air showers. Detailed reviews on the subtleties involved may be found in Refs. 20, 21, 22]. Here we recall only the two main steps, stressing the model dependence involved.

The first step concerns the determination of the proton-air production cross section, namely, the "inelastic cross section in which at least one new hadron is produced in addition to nuclear fragments" [21]. This is obtained by the formulas

$$
\sigma_{p \text {-air }}^{\text {prod }}(\mathrm{mb})=\frac{2.4 \times 10^{4}}{\lambda_{p-a i r}\left(\mathrm{~g} / \mathrm{cm}^{2}\right)}, \quad \lambda_{p-a i r}\left(\mathrm{~g} / \mathrm{cm}^{2}\right)=\frac{\lambda_{a t t}}{k}
$$

where $\lambda_{p \text {-air }}$ is the interaction length of protons in the atmosphere, $\lambda_{a t t}$ is the shower attenuation length, and the inelasticity coefficient $k$ is a measure of the dissipation of energy through the shower. $\lambda_{\text {att }}$ is an experimental quantity determined through the $\chi_{\max }$ attenuation method or the zenith angle attenuation technique [23]. On the other hand, the coefficient $k$ is model dependent and obtained through Monte Carlo simulation; roughly $k \approx 1.5 \rightarrow \approx 1(k>1)$ when going from Feynman scaling models to strongly scaling violation models in the fragmentation region [21]. 
In a second step $\sigma_{t o t}^{p p}$ is obtained from $\sigma_{p \text {-air }}^{\text {prod }}$ through the multiple diffraction formalism (MDF) by Glauber and Matthiae [24], and taking into account the different processes and effects in the p-air total cross section:

$$
\sigma_{p-a i r}^{t o t}=\sigma_{p-a i r}^{p r o d}+\sigma_{p-a i r}^{e l}+\sigma_{p-a i r}^{q-e l}+\Delta \sigma
$$

where $\sigma_{p-a i r}^{q-e l}$ concerns the quasielastic excitation of the nucleus and $\Delta \sigma$ is the Gribov screening correction due to multiple scattering [25].

Two important inputs at this point are the nucleon distribution function and a relation between the slope parameter $B$ and $\sigma_{t o t}^{p p}$, necessary in the parametrization of the scattering amplitude:

$$
F_{p p}(s, t) \propto \sigma_{t o t}^{p p} \exp \left\{\frac{B(s) t}{2}\right\} .
$$

With the screening correction, the MDF allows the determination of all the above cross sections and from the strong correlation between $\sigma_{p-a i r}^{p r o d}-\sigma_{t o t}^{p p}-B$ the $p p$ total cross section may eventually be estimated (for more details, see 21, 22]).

The results presently available from cosmic-ray experiments, in the above energy region, are characterized by discrepancies, mainly due to the model dependence of $k$ and $B(s)$. Before reviewing these results, it is important to draw attention to two facts. First, as is well known, discrepancies also characterize some $\sigma_{t o t}$ data from accelerator experiments at the highest energies, for example, at $541-546 \mathrm{GeV}$ and mainly at $1.8 \mathrm{TeV}$. Second, when performing quantitative analyses, it is fundamental to select as complete a set of information as possible, or at least those obtained in similar circumstances or bases. In the case of cosmic-ray information, it is important to stress that there is no experimental determination of $\sigma_{t o t}^{p p}$, since all the results are model dependent. Therefore they cannot be distinguished in terms of an "experimental" status and all the information available must be considered.

Despite the model dependence involved, we can classify the complete set of cosmic-ray information available according to the inputs or procedures used and, simultaneously, by the value of the total cross section extracted, as explained in what follows.

On one hand the results usually quoted in the literature concern those obtained by the Fly's Eye Collaboration in 1984 [26] and Akeno (AGASA) Collaboration in 1993 [27]. In the first case, the authors used $k=1.6$ (Feynman scaling model), a relation of proportionality between $B$ and $\sigma_{t o t}^{p p}$, namely, the geometrical scaling model [28], and a Gaussian profile distribution for the nucleus. At $\sqrt{s}=30 \mathrm{TeV}$, from the value $\sigma_{p \text {-air }}^{\text {prod }}=540 \pm 50 \mathrm{mb}$, they extracted $\sigma_{\text {tot }}^{p p}=120 \pm 15 \mathrm{mb}$ [26]. The Akeno Collaboration used $k=1.5$ (Feynman scaling model) and the Durand and Pi approach [29] to extract the proton-proton cross sections in the region $\sqrt{s} \sim 6-20 \mathrm{TeV}$. We shall return to these results later on.

On the other hand, in 1987 Gaisser, Sukhatme, and Yodh (GSY) making use of the Fly's Eye result for $\sigma_{p-a i r}^{\text {prod }}$, as an estimate of the lowest allowed values for $\sigma_{t o t}^{p p}$, and the Chou-Yang prescription for $B(s)$ [31] obtained $\sigma_{p p}^{t o t}=175_{-27}^{+40}$ $\mathrm{mb}$ at $\sqrt{s}=40 \mathrm{TeV}$ 22]. In 1993, Nikolaev claimed that the Akeno results should be corrected in order to take into account the differences between absorption and inelastic cross sections, leading to an increase of the results by $\approx 30$ $\mathrm{mb}$ [32]. The analysis by Nikolaev was also motivated by previous results from a QCD model of the Pomeron [33].

At this point some fundamental facts concerning all these results must be stressed. First, as reviewed above, the Fly's Eye and Akeno results for $\sigma_{t o t}^{p p}$ are as model dependent as those by Nikolaev and GSY and therefore cannot be considered as experimental results; what can and must be discussed is the class of model used in each case. In this sense, we first note that since the Fly's Eye Collaboration used the geometrical scaling hypothesis, which is violated even at the collider energy, their result is probably wrong. Second, in 1990, Durand and Pi asserted in Ref. 30 that their results published in 1988 [29], and used by the Akeno Collaboration [27], should be disregarded due to a wrong approximation concerning fluctuations. The new results presented in [30] (Fig. 11 in that paper) introduced significant changes in the scenario. For example, for $\sigma_{p-a i r} \approx 550 \mathrm{mb}$, the Akeno result with the method by Durand and $\mathrm{Pi}$ [29] was $\sigma_{\text {tot }}^{p p}=124 \mathrm{mb}$ and from Fig. 11 in Ref. [30] the value extracted is $\sigma_{t o t}^{p p}=137 \mathrm{mb}$, that is, an increase of $\approx 11 \%$; to the Fly's Eye published point [26], the corresponding value is $\sigma_{t o t}^{p p}=135 \mathrm{mb}[30]$, that is, an increase of $\approx 13 \%$. However, to our knowledge no other publication by Durand and Pi on the subject appeared in the literature. Finally, it should be stressed that the Chou-Yang prescription, used by Gaisser, Sukhatme, and Yodh, is somewhat model independent and, also to our knowledge, the results by these authors together with those by Nikolaev have never been criticized, despite the intrinsic model dependence involved. In conclusion, we understand that all these facts, not usually discussed in the literature, suggest an increase of the total cross section faster than indicated by the Akeno and Fly's Eye results and that this indication has a reasonable basis.

It should also be recalled that, more recently, Block, Halzen, and Stanev (BHS) obtained estimations for $\sigma_{t o t}^{p p}$

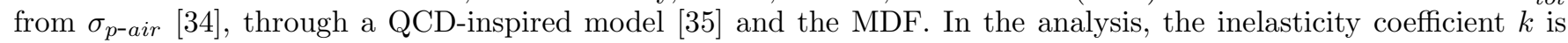


TABLE I: Estimations for $\sigma_{\text {tot }}^{p p}(s)$ (in mb) from cosmic-ray data [19].

\begin{tabular}{|c|c|c|c|c|c|c|}
\hline $\begin{array}{c}E_{l a b} \\
\left(10^{16} \mathrm{eV}\right)\end{array}$ & $\begin{array}{c}\sqrt{s} \\
(\mathrm{TeV}) \\
\end{array}$ & $\begin{array}{c}\text { Akeno (AGASA) } \\
{[27]}\end{array}$ & $\begin{array}{c}\text { Fly's Eye } \\
{[26]}\end{array}$ & $\begin{array}{c}\text { Nikolaev } \\
{[32]}\end{array}$ & $\begin{array}{r}\text { GSY } \\
{[22]} \\
\end{array}$ & $\begin{array}{c}\text { BHS } \\
{[37]} \\
\end{array}$ \\
\hline 2.02 & 6.2 & $93 \pm 14$ & - & $120 \pm 15$ & - & $91 \pm 15$ \\
\hline 3.52 & 8.1 & $101 \pm 16$ & - & $130 \pm 18$ & - & $100 \pm 18$ \\
\hline 6.11 & 10.6 & $117 \pm 18$ & - & $154 \pm 17$ & - & $118 \pm 17$ \\
\hline 10.63 & 14.0 & $104 \pm 26$ & - & $135 \pm 29$ & - & $103 \pm 29$ \\
\hline 18.47 & 18.4 & $100 \pm 27$ & - & $129 \pm 30$ & - & $99 \pm 30$ \\
\hline 32.09 & 24.3 & $124 \pm 34$ & - & $162 \pm 38$ & - & $124 \pm 37$ \\
\hline 47.96 & 30.0 & - & $120 \pm 15$ & - & - & $103 \pm 22$ \\
\hline 85.26 & 40.0 & - & - & - & $175 \pm 34$ & - \\
\hline
\end{tabular}

considered as a free parameter, determined through a global fit, including $p p$ and $\bar{p} p$ accelerator data and the published results on the $p$-air cross section [34]. Specifically, $k$ is determined in a way that their predictions for $\sigma_{t o t}^{p p}=\sigma_{t o t}^{\bar{p} p}$ (asymptotically) matches $\sigma_{p \text {-air }}$ through the MDF. The resulting value $k \approx 1.35$ seems to be in accord with those obtained by combinations of different simulation programs, namely, $k \approx 1.15-1.30[36]$. The extracted $\sigma_{\text {tot }}^{p p}(s)$ at the cosmic-ray energies shows agreement with the Akeno results and is about $17 \mathrm{mb}$ below the Fly's Eye value at 30 TeV.

The numbers associated with all the above cosmic-ray estimations of $\sigma_{\text {tot }}^{p p}$ are displayed in Table 1 . Despite the importance and originality of the approach by BHS, we shall not include it in our analysis, because they are in agreement with the Akeno results (Table 1) that will be used. However we will treat this case in further work. With the exception of the BHS results, all the other cosmic-ray estimates discussed above together with the accelerator results are displayed in Fig. 1.

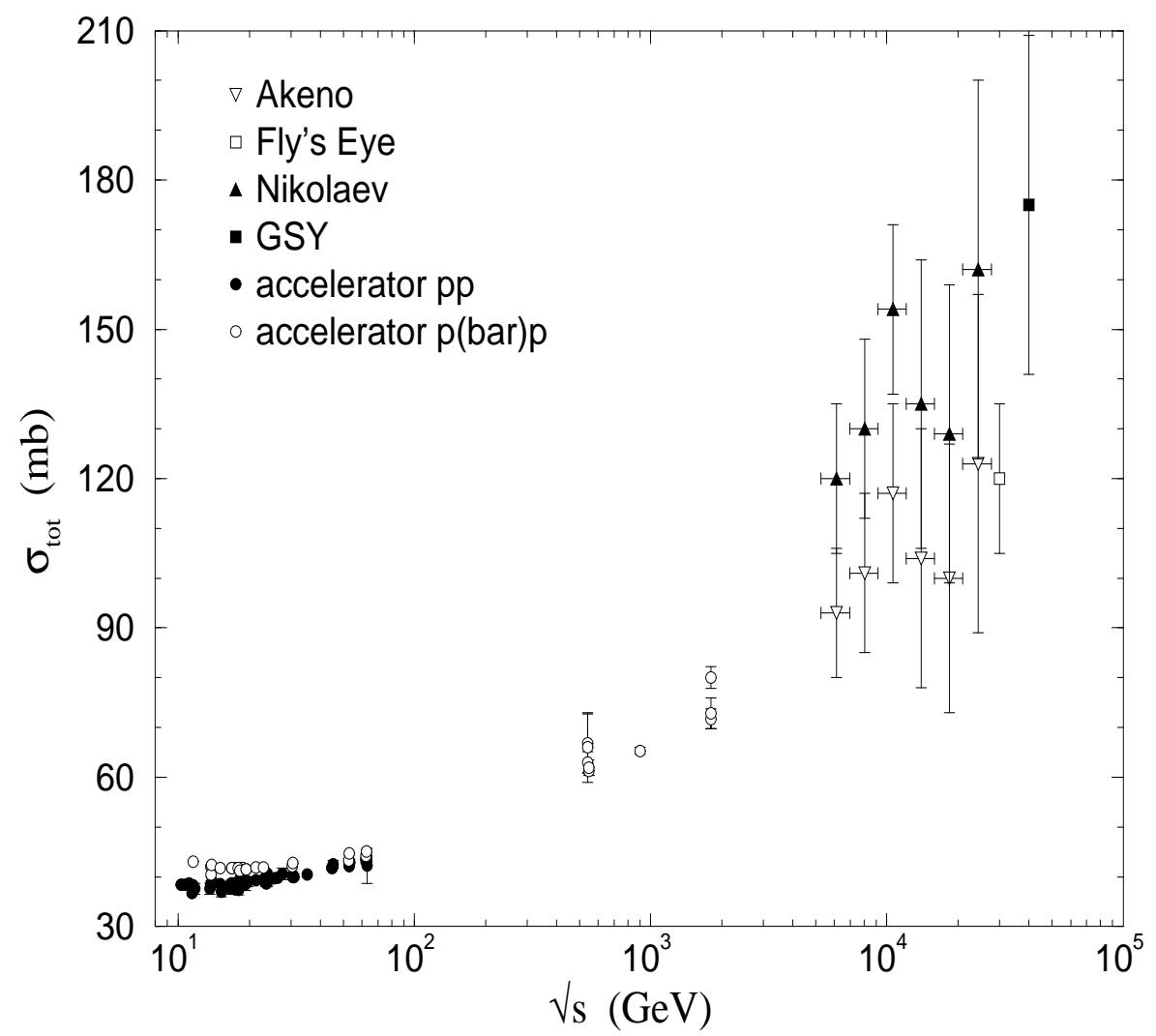

FIG. 1: Total cross sections ( $p p$ and $\bar{p} p$ above $\sqrt{s}=10 \mathrm{GeV}$ ): accelerator data and cosmic-ray information available (Table I). 


\section{Ensembles}

From Fig. 1 we see that, despite the large error bars in the cosmic-ray region, we can identify two distinct set of estimations: one represented by the results of the Fly's Eye Collaboration together with those by the Akeno Collaboration; the other by the results of Gaisser, Sukhatme, and Yodh with those by Nikolaev, which follow the higher estimates by Durand and Pi 30]. Taken separately these two sets suggest different scenarios for the increase of the total cross section, as already claimed before [16, 17, 38].

Based on these considerations, we shall investigate the behavior of the total cross section by taking into account the discrepancies that characterize the cosmic ray information and, to this end, we shall consider two ensembles of data and experimental information with the following notation:

- Ensemble I: $\bar{p} p$ accelerator data and $p p$ accelerator data + Akeno + Fly's Eye;

- Ensemble II: $\bar{p} p$ accelerator data and $p p$ accelerator data + Nikolaev + GSY.

To some extent, ensemble I represents a kind of high-energy standard picture and ensemble II a nonstandard one. However, the consistence among the results by Nikolaev and GSY must be stressed, in addition to their agreement with the last results by Durand and Pi (Fig. 11 in Ref. [30]). We add also that, from the discussion in Sec. II B, both ensembles seem equally probable.

\section{ANALYTICAL APPROACH}

In this section we first review the essential formulas in the derivative dispersion approach and recall some fundamental theorems and high-energy bounds. The parametrizations that characterize the analytical models to be used in the next section are also presented.

\section{A. Analyticity relations}

For $p p$ and $\bar{p} p$ scattering, analyticity and crossing symmetry allow us to connect $\sigma_{\text {tot }}(s)$ and $\rho(s)$ through two compact and symmetric formulas:

$$
\begin{aligned}
& \rho^{p p} \sigma_{t o t}^{p p}(s)=E\left(\sigma_{+}\right)+O\left(\sigma_{-}\right), \\
& \rho^{\bar{p} p} \sigma_{t o t}^{\bar{p} p}(s)=E\left(\sigma_{+}\right)-O\left(\sigma_{-}\right),
\end{aligned}
$$

where

$$
\sigma_{ \pm}(s)=\frac{\sigma_{t o t}^{p p} \pm \sigma_{t o t}^{\bar{p} p}}{2}
$$

and $E\left(\sigma_{+}\right), O\left(\sigma_{-}\right)$are analytic transforms relating the real and imaginary parts of crossing even and odd amplitudes, respectively. These analyticity relations are usually expressed in an integral form and in the case of the forward direction the standard once subtracted integral dispersion relations may be expressed by [5, 7]

$$
\begin{gathered}
E_{\text {int }}\left(\sigma_{+}\right) \equiv \frac{K}{s}+\frac{2 s}{\pi} \int_{s_{o}}^{\infty} \mathrm{d} s^{\prime}\left[\frac{1}{s^{2}-s^{2}}\right] \sigma_{+}\left(s^{\prime}\right)=\frac{\operatorname{Re} F_{+}(s)}{s}, \\
O_{i n t}\left(\sigma_{-}\right) \equiv \frac{2}{\pi} \int_{s_{o}}^{\infty} \mathrm{d} s^{\prime}\left[\frac{s^{\prime}}{s^{\prime 2}-s^{2}}\right] \sigma_{-}\left(s^{\prime}\right)=\frac{\operatorname{Re} F_{-}(s)}{s}
\end{gathered}
$$

where $K$ is the subtraction constant. Since we shall be interested in the high-energy region, we used in the above formulas the c.m. energy, instead of the laboratory energy and momentum [5, 7].

At sufficiently high energies integral analyticity relations may be replaced by derivative forms, usually called analyticity relations, which are more useful for practical calculations. In these formulas, differentiation with respect to 
the logarithm of the energy occurs in the argument of a trigonometric operator expressed by its series [10, 11, 12]. Here we shall use the standard form deduced by Bronzan, Kane, and Sukhatme [11] (see also [16, 39]), obtained from the integral form in the high-energy limit:

$$
\begin{gathered}
E_{d e r}\left(\sigma_{+}\right) \equiv \frac{K}{s}+\tan \left[\frac{\pi}{2} \frac{d}{d \ln s}\right] \sigma_{+}(s)=\frac{\operatorname{Re} F_{+}(s)}{s}, \\
O_{d e r}\left(\sigma_{-}\right) \equiv \tan \left[\frac{\pi}{2}\left(1+\frac{d}{d \ln s}\right)\right] \sigma_{-}(s)=\frac{\operatorname{Re} F_{-}(s)}{s} .
\end{gathered}
$$

Operationally these transforms may be evaluated through the expansions

$$
\begin{aligned}
E_{d e r}\left(\sigma_{+}\right) & -\frac{K}{s}=\left[\frac{\pi}{2} \frac{d}{d \ln s}+\frac{1}{3}\left(\frac{\pi}{2} \frac{d}{d \ln s}\right)^{3}+\frac{2}{5}\left(\frac{\pi}{2} \frac{d}{d \ln s}\right)^{5}+\ldots\right] \sigma_{+}(s), \\
O_{d e r}\left(\sigma_{-}\right) & =-\int\left\{\frac{d}{d \ln s}\left[\cot \left(\frac{\pi}{2} \frac{d}{d \ln s}\right)\right] \sigma_{-}(s)\right\} d \ln s \\
& =-\frac{2}{\pi} \int\left\{\left[1-\frac{1}{3}\left(\frac{\pi}{2} \frac{d}{d \ln s}\right)^{2}-\frac{1}{45}\left(\frac{\pi}{2} \frac{d}{d \ln s}\right)^{4}-\ldots\right] \sigma_{-}(s)\right\} d \ln s .
\end{aligned}
$$

This completes the analytical approach: with an input parametrization for $\sigma_{\text {tot }}(s)$, Eqs. (1)-(4) allow, in principle, the determination of $\rho(s)$, by means of either the integral forms Eqs. (5)-(6) or the derivative (analyticity) ones Eqs. (7)-(8).

\section{B. Theorems at high energies}

For future reference, we briefly recall here two rigorous high-energy results for the asymptotic behavior of the cross sections. The Froissart-Martin bound states that as $s \rightarrow \infty$ [40, 41]

$$
\sigma_{t o t} \leq C \ln ^{2} s
$$

and, according to the generalized form of the Pomeranchuk theorem [3, 42], if the Froissart-Martin bound is reached the difference between $\bar{p} p$ and $p p$ total cross sections goes as

$$
\Delta \sigma_{t o t}=\sigma_{t o t}^{p \bar{p}}-\sigma_{t o t}^{p p} \leq c \frac{\sigma_{t o t}^{\bar{p} p}+\sigma_{t o t}^{p p}}{\ln s}
$$

which means that the difference may increase as $\ln s$. From Eq. (4), this difference is given by the crossing odd component

$$
\Delta \sigma_{t o t}(s)=-2 \sigma_{-}(s)
$$

and therefore, if the Froissart-Martin bound is saturated, a rigorous result is that, asymptotically, the difference $\Delta \sigma_{t o t}(s)$ is controlled by the odd component and the maximum contribution is given by $\sigma_{-}^{\max }(s)=\ln s$. This corresponds to one of the variants of the Odderon picture [12, 43] and the increase as $\ln s$ is the maximum Odderon hypothesis. If the odd contribution in the imaginary part of the amplitude is not present at the highest energies, then $\Delta \sigma_{\text {tot }}=0$. The possible effects in the real part will be discussed in what follows.

\section{Analytic models}

In the formulas that follows we denote $s / s^{\prime}$ with $s^{\prime}=1 \mathrm{GeV}^{2}$ by $s$. We shall consider two different parametrizations for the total cross section, the main difference being the asymptotic limits, which allow the dominance of an even or odd amplitude. 


\section{Donnachie-Landshoff}

The Donnachie-Landshoff (DL) parametrizations for the total cross sections are expressed by 18

$$
\sigma_{\text {tot }}^{p p}(s)=X s^{\epsilon}+Y s^{-\eta}, \quad \sigma_{\text {tot }}^{\bar{p} p}(s)=X s^{\epsilon}+Z s^{-\eta},
$$

where, originally, $X=21.7 \mathrm{mb}, Y=56.08 \mathrm{mb}, Z=98.39 \mathrm{mb}, \epsilon=0.0808$, and $\eta=0.4525$. We observe that this model predicts that the difference between the two cross sections is given by

$$
\Delta \sigma=\sigma_{t o t}^{\bar{p} p}(s)-\sigma_{t o t}^{p p}(s)=(Z-Y) s^{-\eta} \rightarrow 0 \quad \text { (asymptotically). }
$$

Through the formalism described in Sec. III A, substitution of the parametrizations (9) into Eq. (4) and then in Eqs. (5)-(6) with $s_{0}=0$ or Eqs. (7)-(8) gives

$$
\begin{gathered}
E\left(\sigma_{+}\right)=\frac{K}{s}+\left[X \tan \left(\frac{\pi \epsilon}{2}\right)\right] s^{\epsilon}-\left[\frac{(Y+Z)}{2} \tan \left(\frac{\pi \eta}{2}\right)\right] s^{\eta}, \\
O\left(\sigma_{-}\right)=\left[\frac{(Y-Z)}{2} \cot \left(\frac{\pi \eta}{2}\right)\right] s^{\eta},
\end{gathered}
$$

and from Eqs. (2)-(3) we obtain the analytical expressions for $\rho(s)$ :

$$
\begin{aligned}
& \rho^{p p}(s)=\frac{1}{\sigma_{t o t}^{p p}(s)}\left\{\frac{K}{s}+\left[X \tan \left(\frac{\pi \epsilon}{2}\right)\right] s^{\epsilon}+\left[\frac{(Y-Z)}{2} \cot \left(\frac{\pi \eta}{2}\right)-\frac{(Y+Z)}{2} \tan \left(\frac{\pi \eta}{2}\right)\right] s^{-\eta}\right\}, \\
& \rho^{\bar{p} p}(s)=\frac{1}{\sigma_{t o t}^{\bar{p} p}(s)}\left\{\frac{K}{s}+\left[X \tan \left(\frac{\pi \epsilon}{2}\right)\right] s^{\epsilon}+\left[\frac{(Z-Y)}{2} \cot \left(\frac{\pi \eta}{2}\right)-\frac{(Y+Z)}{2} \tan \left(\frac{\pi \eta}{2}\right)\right] s^{-\eta}\right\} .
\end{aligned}
$$

Since in this model $\Delta \sigma \rightarrow 0$ asymptotically, for $\sigma_{t o t}^{\bar{p} p}=\sigma_{t o t}^{p p} \equiv \sigma_{t o t}$ we have

$$
\Delta \rho=\rho^{\bar{p} p}-\rho^{p p} \sim \frac{1}{\sigma_{t o t}(s)}(Z-Y) \cot \left(\frac{\pi \eta}{2}\right) s^{-\eta} \quad \rightarrow \quad 0 \quad \text { as } \quad s \quad \rightarrow \quad \infty .
$$

\section{Kang-Nicolescu}

The parametrizations for the total cross sections used by Kang and Nicolescu (KN) under the hypothesis of the Odderon [43] are expressed by [12]

$$
\sigma_{t o t}^{p p}(s)=A_{1}+B_{1} \ln s+k \ln ^{2} s, \quad \sigma_{t o t}^{\bar{p} p}(s)=A_{2}+B_{2} \ln s+k \ln ^{2} s+2 R s^{-1 / 2} .
$$

Differently from the previous case, this model predicts that the difference between the two cross sections is given by

$$
\begin{aligned}
\Delta \sigma=\sigma_{\text {tot }}^{\bar{p} p}(s) & -\sigma_{\text {tot }}^{p p}(s)=\left(A_{2}-A_{1}\right)+\left(B_{2}-B_{1}\right) \ln s+2 R s^{-1 / 2} \\
& \rightarrow \Delta A+\Delta B \ln s \quad \text { (asymptotically) }
\end{aligned}
$$

so that, if $\Delta A \neq 0$ and/or $\Delta B \neq 0$, the total cross section difference may increase and $\sigma_{\text {tot }}^{p p}$ may even become greater than $\sigma_{t o t}^{\vec{p} p}$, depending on the values and signs of $\Delta A$ and $\Delta B$, which is formally in agreement with the theorems of Sec. III B.

With a similar procedure as in the previous model the use of the analyticity relations (7)-(8) leads to 


$$
\begin{gathered}
E\left(\sigma_{+}\right)=\frac{K}{s}+\frac{\pi}{2}\left(\frac{B_{1}+B_{2}}{2}\right)+\pi k \ln s-R s^{-1 / 2}, \\
O\left(\sigma_{-}\right)=\left(\frac{A_{2}-A_{1}}{\pi}\right) \ln s+\left(\frac{B_{2}-B_{1}}{2 \pi}\right) \ln ^{2} s-R s^{-1 / 2},
\end{gathered}
$$

and from Eqs. (2)-(3)

$$
\begin{gathered}
\rho^{p p}=\frac{1}{\sigma_{\text {tot }}^{p p}}\left\{\frac{K}{s}+\frac{\pi}{2}\left(\frac{B_{1}+B_{2}}{2}\right)+\left(\pi k+\frac{A_{2}-A_{1}}{\pi}\right) \ln s+\left(\frac{B_{2}-B_{1}}{2 \pi}\right) \ln ^{2} s-2 R s^{-1 / 2}\right\}, \\
\rho^{\bar{p} p}=\frac{1}{\sigma_{\text {tot }}^{\bar{p} p}}\left\{\frac{K}{s}+\frac{\pi}{2}\left(\frac{B_{1}+B_{2}}{2}\right)+\left(\pi k-\frac{A_{2}-A_{1}}{\pi}\right) \ln s-\left(\frac{B_{2}-B_{1}}{2 \pi}\right) \ln ^{2} s\right\} .
\end{gathered}
$$

In this case, if $\Delta A$ and $\Delta B$ are sufficiently small, so that we may replace $\sigma_{t o t}^{\bar{p} p} \approx \sigma_{t o t}^{p p} \equiv \sigma_{t o t}(s)$, then, asymptotically,

$$
\Delta \rho=\rho^{\bar{p} p}-\rho^{p p} \sim-\frac{1}{\pi \sigma_{t o t}(s)}\left\{\Delta A \ln s+\Delta B \ln ^{2} s\right\} .
$$

This means that, depending on the fit results, there may be a change of sign in $\Delta \rho$, with $\rho^{p p}$ becoming greater than $\rho^{\bar{p} p}$ at some finite energy.

All these possibilities for a change of sign in the differences between $\bar{p} p$ and $p p$ total cross sections and/or the $\rho$ parameters are based on the concept of the Odderon [43]; the case of a change of sign in $\Delta \sigma_{\text {tot }}(s)$ was early discussed by Bernard, Gauron, and Nicolescu [44] and that in $\Delta \rho$ by Gauron, Nicolescu, and Leader [45]. They are associated with the condition that the maximal odderon dominates the imaginary or real part of the amplitude, respectively.

\section{FITTING OF THE DATA AND RESULTS}

In order to investigate all the points raised in Sec. I and their possible interconnections, we perform 16 different fits through the program CERN-MINUIT. In these fits we use both ensembles I and II defined in Sec. II and both the DL and KN models described in Sec. III. For each of these four possibilities we perform global and individual fits to $\sigma_{\text {tot }}$ and $\rho$ and, in each case, we either consider the subtraction constant $K$ as a free fit parameter or assume $K=0$.

In the individual approach we first fit only the total cross section data and then extract the corresponding $\rho(s)$ in the case of $K=0$, or, with the results for $\sigma_{\text {tot }}(s)$, we fit only the $\rho$ data with $K$ as a free fit parameter.

The numerical results of the fits and statistical information are all displayed in Tables II and III for the DL and KN models, respectively. The corresponding curves together with the experimental data are shown in Figs. 2, 3, and 4 for the DL model and Figs. 5, 6, and 7 for the KN model. 
TABLE II: Results with the Donnachie-Landshoff model.

\begin{tabular}{|c|c|c|c|c|c|c|c|c|}
\hline \multirow{3}{*}{$\begin{array}{c}\text { Fit: } \\
\text { Quantity: } \\
\text { Ensemble: }\end{array}$} & \multicolumn{4}{|c|}{ Individual } & \multirow{2}{*}{\multicolumn{4}{|c|}{$\begin{array}{c}\text { Global } \\
\sigma_{\text {tot }} \text { and } \rho\end{array}$}} \\
\hline & \multicolumn{2}{|c|}{$\sigma_{t o t}$} & \multicolumn{2}{|c|}{$\rho$} & & & & \\
\hline & I & II & $\mathrm{I}$ & II & I & II & I & II \\
\hline No. of points & 102 & 102 & 63 & 63 & 165 & 165 & 165 & 165 \\
\hline$\chi^{2} / \mathrm{DOF}$ & 0.76 & 0.96 & 1.55 & 1.84 & 1.09 & 1.24 & 0.84 & 0.98 \\
\hline$X(\mathrm{mb})$ & $20.0 \pm 0.7$ & $19.3 \pm 0.7$ & - & - & $21.6 \pm 0.4$ & $21.2 \pm 0.4$ & $21.4 \pm 0.4$ & $21.1 \pm 0.4$ \\
\hline$Y(\mathrm{mb})$ & $48 \pm 5$ & $46 \pm 5$ & - & - & $51 \pm 3$ & $51 \pm 3$ & $67 \pm 6$ & $67 \pm 5$ \\
\hline$Z(\mathrm{mb})$ & $74 \pm 10$ & $70 \pm 9$ & - & - & $85 \pm 5$ & $84 \pm 5$ & $114 \pm 11$ & $112 \pm 11$ \\
\hline$\eta$ & $0.37 \pm 0.03$ & $0.35 \pm 0.03$ & - & - & $0.43 \pm 0.02$ & $0.42 \pm 0.02$ & $0.48 \pm 0.02$ & $0.47 \pm 0.02$ \\
\hline$\epsilon$ & $0.088 \pm 0.003$ & $0.091 \pm 0.003$ & - & - & $0.081 \pm 0.002$ & $0.083 \pm 0.002$ & $0.083 \pm 0.002$ & $0.084 \pm 0.002$ \\
\hline$K$ & - & - & $235 \pm 32$ & $245 \pm 32$ & 0 & 0 & $306 \pm 54$ & $307 \pm 52$ \\
\hline Figure: & $2(\mathrm{a})$ ar & d $2(b)$ & $2(\mathrm{a})$ al & d $2(c)$ & : & 3 & 4 & 4 \\
\hline
\end{tabular}
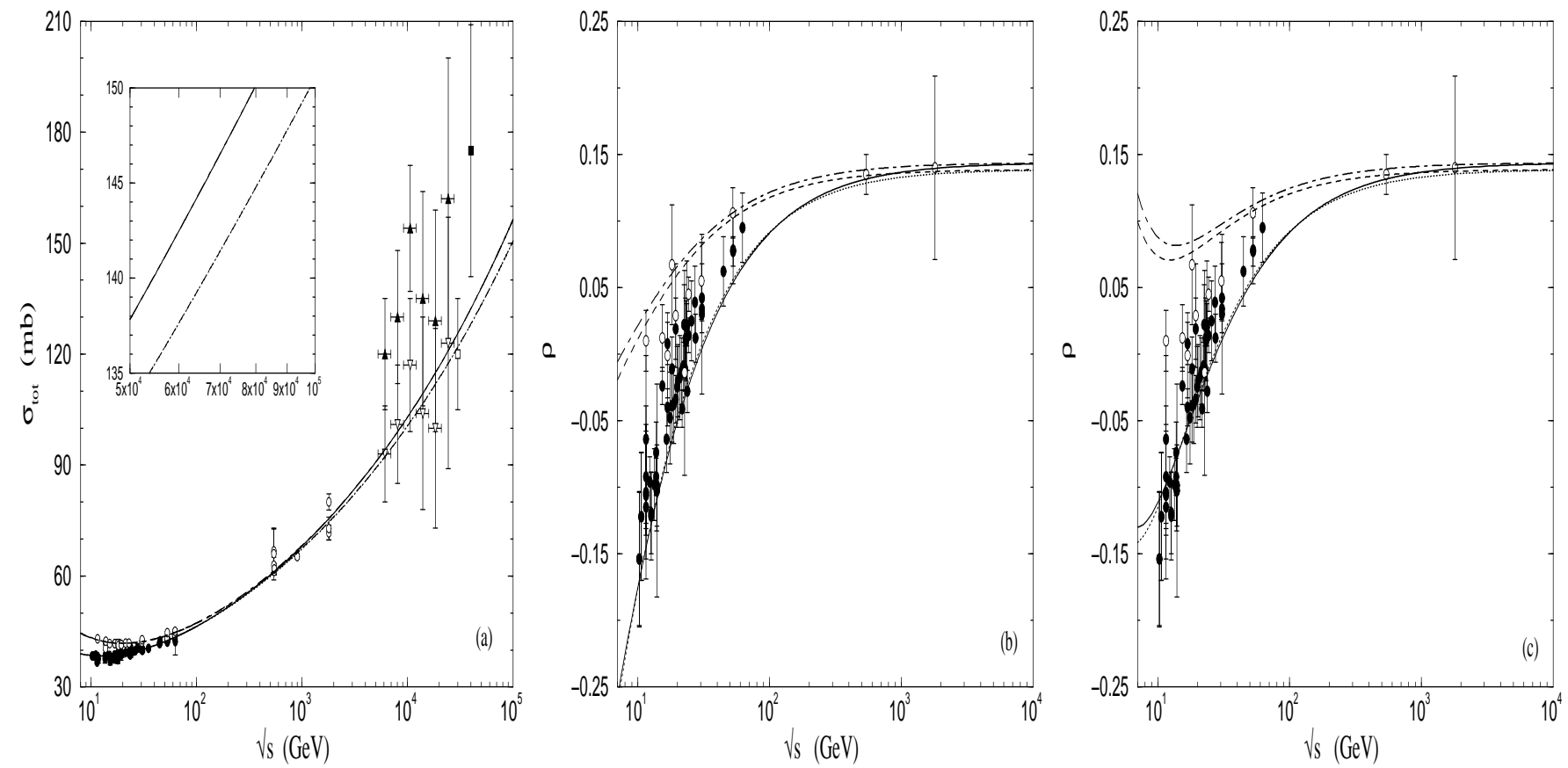

FIG. 2: Fits to $p p$ and $\bar{p} p$ total cross section data from ensembles I (dotted curves for $p p$ and dashed for $\bar{p} p$ ) and II (solid curves for $p p$ and dot-dashed for $\bar{p} p$ ), through the DL parametrization (a) and the corresponding predictions for $\rho(s)$ with $K=0$ (b) and $K$ as free fit parameter (c) - columns $2-5$ in Table II. 

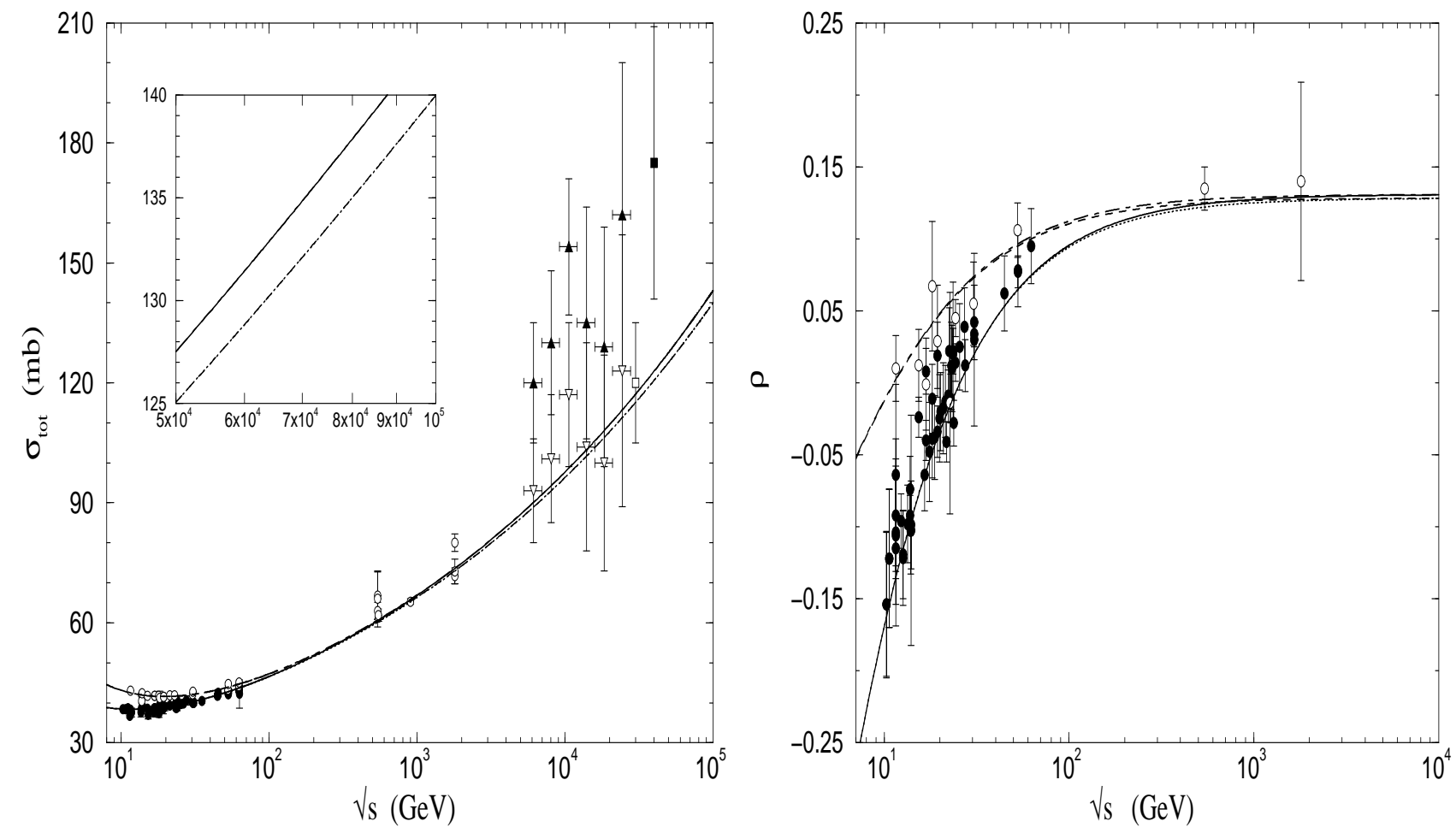

FIG. 3: Simultaneous fits to $\sigma_{\text {tot }}(s)$ and $\rho(s)$ through the DL parametrization with $K=0$ and ensembles I (dotted curves for $p p$ and dashed for $\bar{p} p$ ) and II (solid curves for $p p$ and dot-dashed for $\bar{p} p$ ) — columns 6 and 7 in Table II.
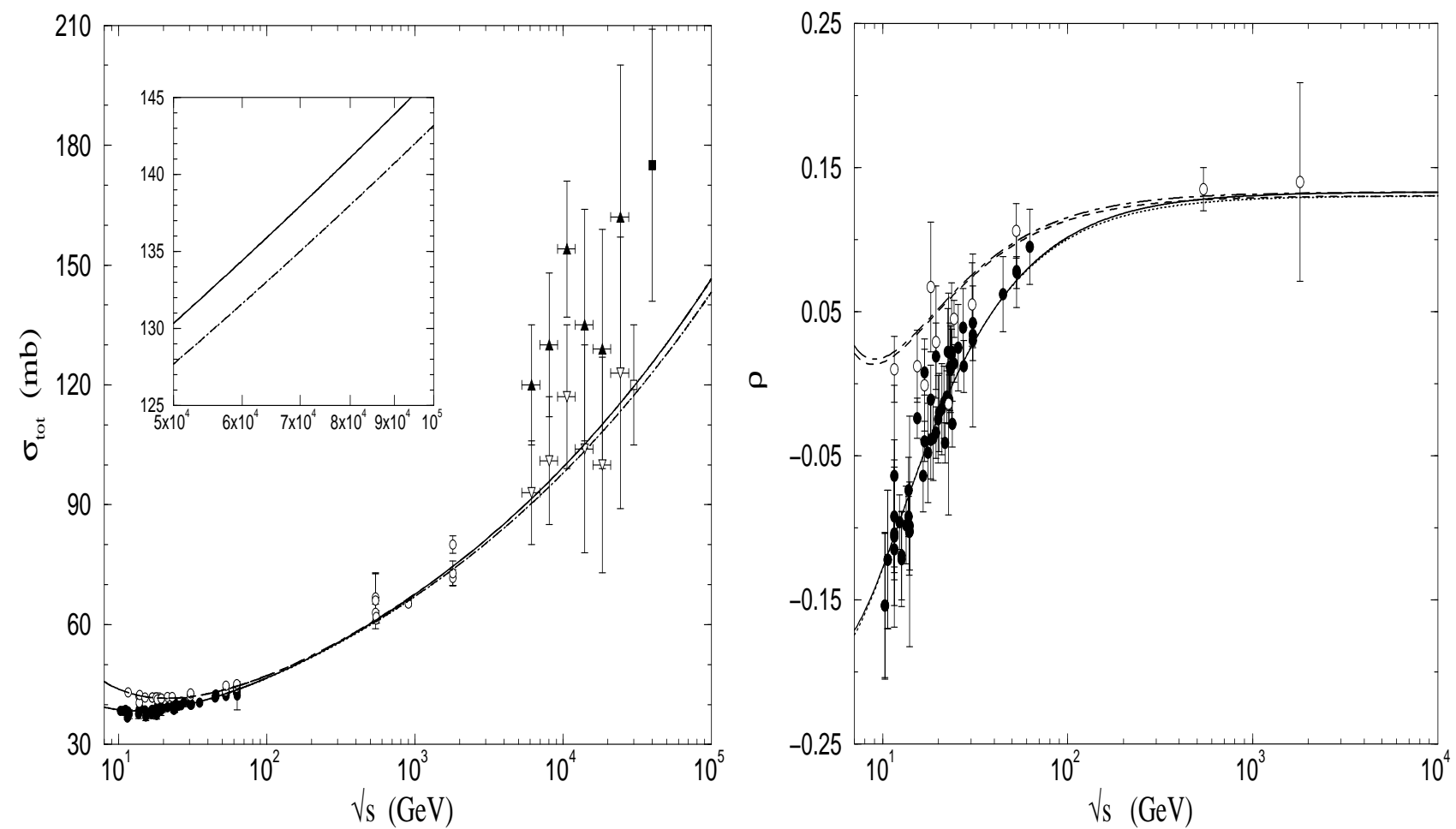

FIG. 4: Simultaneous fits to $\sigma_{\text {tot }}(s)$ and $\rho(s)$ through the DL parametrization with $K$ as free parameter and ensembles I (dotted curves for $p p$ and dashed for $\bar{p} p$ ) and II (solid curves for $p p$ and dot-dashed for $\bar{p} p$ )—columns 8 and 9 in Table II. 
TABLE III: Results with the Kang-Nicolescu model.

\begin{tabular}{|c|c|c|c|c|c|c|c|c|}
\hline \multirow{3}{*}{$\begin{array}{c}\text { Fit: } \\
\text { Quantity: } \\
\text { Ensemble: }\end{array}$} & \multicolumn{4}{|c|}{ Individual } & \multirow{2}{*}{\multicolumn{4}{|c|}{$\begin{array}{c}\text { Global } \\
\sigma_{\text {tot }} \text { and } \rho\end{array}$}} \\
\hline & \multicolumn{2}{|c|}{$\sigma_{t o t}$} & \multicolumn{2}{|c|}{$\rho$} & & & & \\
\hline & I & II & I & II & I & II & $\mathrm{I}$ & II \\
\hline No. of points & 102 & 102 & 63 & 63 & 165 & 165 & 165 & 165 \\
\hline$\chi^{2} / \mathrm{DOF}$ & 0.78 & 0.91 & 1.05 & 35.2 & 0.78 & 0.87 & 0.77 & 0.86 \\
\hline$A_{1}(\mathrm{mb})$ & $44 \pm 1$ & $47 \pm 1$ & - & - & $44.7 \pm 0.6$ & $45.2 \pm 0.6$ & $44.4 \pm 0.6$ & $45.0 \pm 0.7$ \\
\hline$A_{2}(\mathrm{mb})$ & $45 \pm 3$ & $52 \pm 3$ & - & - & $44.7 \pm 0.7$ & $45.2 \pm 0.7$ & $44.5 \pm 0.7$ & $45.1 \pm 0.7$ \\
\hline$B_{1}(\mathrm{mb})$ & $-2.9 \pm 0.3$ & $-3.6 \pm 0.4$ & - & - & $-3.0 \pm 0.2$ & $-3.1 \pm 0.2$ & $-2.9 \pm 0.2$ & $-3.1 \pm 0.2$ \\
\hline$B_{2}(m b)$ & $-2.9 \pm 0.6$ & $-4.2 \pm 0.6$ & - & - & $-2.9 \pm 0.2$ & $-3.1 \pm 0.2$ & $-2.9 \pm 0.2$ & $-3.1 \pm 0.2$ \\
\hline$k(\mathrm{mb})$ & $0.33 \pm 0.03$ & $0.39 \pm 0.03$ & - & - & $0.34 \pm 0.01$ & $0.35 \pm 0.01$ & $0.33 \pm 0.01$ & $0.35 \pm 0.01$ \\
\hline$R(\mathrm{mb})$ & $24 \pm 7$ & $12 \pm 7$ & - & - & $25.9 \pm 0.8$ & $25.9 \pm 0.8$ & $25.3 \pm 0.9$ & $25.4 \pm 0.9$ \\
\hline$K$ & - & - & $-198 \pm 32$ & $-1369 \pm 32$ & 0 & 0 & $-72 \pm 46$ & $-63 \pm 46$ \\
\hline Figure: & \multicolumn{2}{|c|}{$5(\mathrm{a})$ and $5(\mathrm{~b})$} & \multicolumn{2}{|c|}{$5(\mathrm{a})$ and $5(\mathrm{c})$} & \multicolumn{2}{|c|}{6} & \multicolumn{2}{|c|}{7} \\
\hline
\end{tabular}
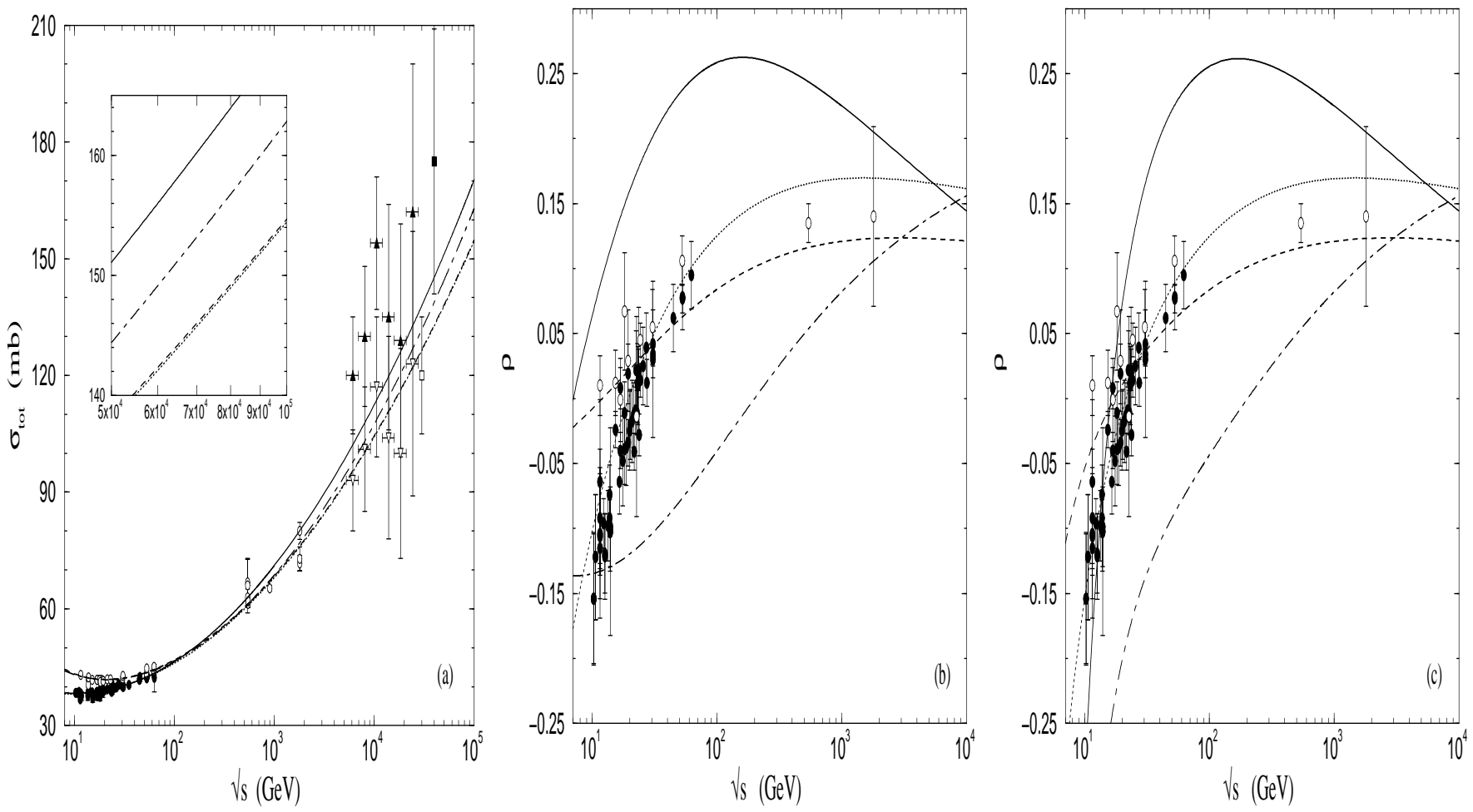

FIG. 5: Fits to $p p$ and $\bar{p} p$ total cross section data from ensembles I (dotted curves for $p p$ and dashed for $\bar{p} p$ ) and II (solid curves for $p p$ and dot-dashed for $\bar{p} p$ ), through the KN parametrization (a) and the corresponding predictions for $\rho(s)$ with $K=0$ (b) and $K$ as free fit parameter (c) - columns 2 and 3 in Table III. 

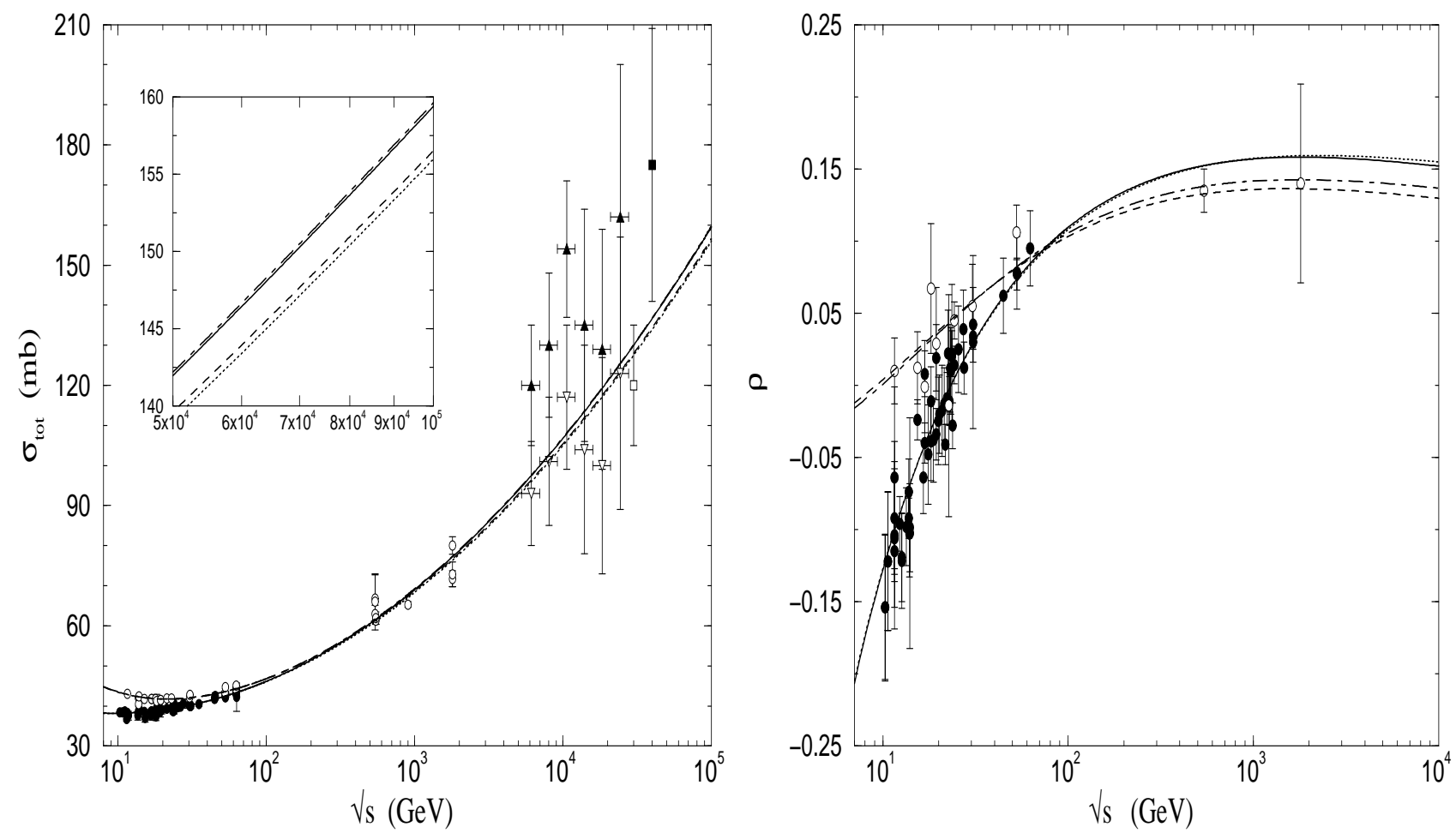

FIG. 6: Simultaneous fits to $\sigma_{\text {tot }}(s)$ and $\rho(s)$ through the KN parametrization with $K=0$ and ensembles I (dotted curves for $p p$ and dashed for $\bar{p} p$ ) and II (solid curves for $p p$ and dot-dashed for $\bar{p} p$ ) — columns 6 and 7 in Table III.
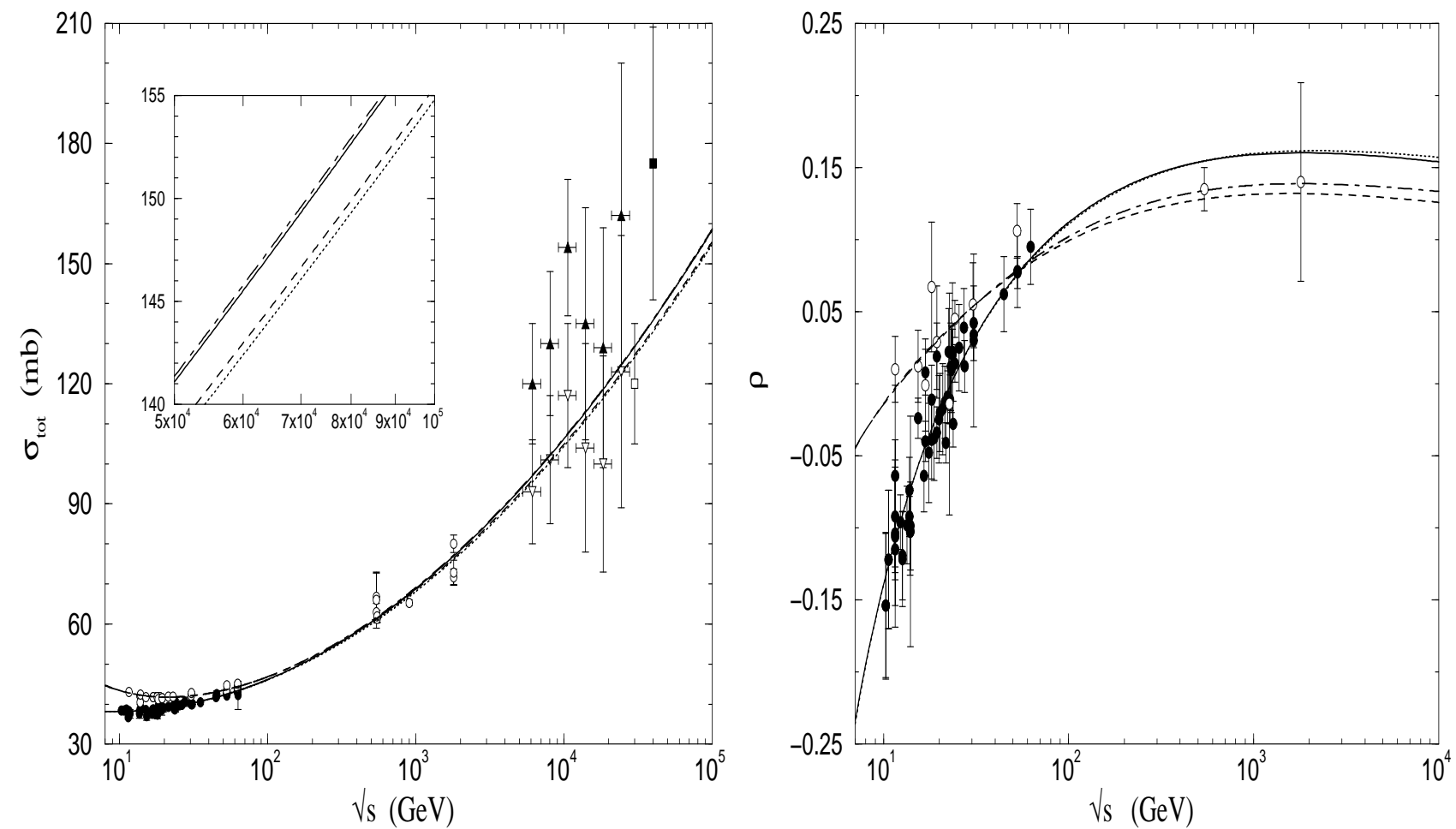

FIG. 7: Simultaneous fits to $\sigma_{\text {tot }}(s)$ and $\rho(s)$ through the KN parametrization with $K$ as free parameter and ensembles I (dotted curves for $p p$ and dashed for $\bar{p} p$ ) and II (solid curves for $p p$ and dot-dashed for $\bar{p} p$ )—columns 8 and 9 in Table III. 


\section{CONCLUSIONS AND FINAL REMARKS}

We shall focus our conclusions and present some discussion on the following five points.

Ensembles I and II. The figures corresponding to fits to the total cross section data show that, in general, the results with ensembles I and II do not differ substantially, except in the case of individual fits with the KN model [Fig. 5.(a)]. Also, the cosmic-ray information in ensemble II (Nikolaev and GSY) is not described in all the cases. This is a consequence of the small number of points and the large error bars in comparison with the accelerator data, as well as the choices for the parametrizations (the models used). However, we must stress that, despite being a nonstandard result, the cosmic-ray information in ensemble II has a reasonable basis, as discussed in Sec. II B. Certainly, more precise data are necessary for a truly conclusive result, but, at present, it seems reasonable and interesting to investigate the semiquantitative consequences of this nonstandard possibility.

Based on these ideas, in what follows, we shall consider the results with both ensembles. However, it must also be noted that some of the conclusions that follow are independent of the ensemble used, such as those concerning global vs individual fits and the role of the subtraction constant.

Bounds for the pomeron intercept. With the DL model and the cosmic-ray information in ensembles I and II, we may infer some novel limit values for the soft Pomeron intercept $\alpha_{\mathbb{P}}(0)=1+\epsilon$. From Table II the highest (lowest) $\epsilon$ value was obtained with ensemble II (I), in the case of individual fits to $\sigma_{\text {tot }}$ (global fits to $\sigma_{\text {tot }}$ and $\rho$ ), namely,

$$
\epsilon_{\text {upper }}=0.094 \quad \text { and } \quad \epsilon_{\text {lower }}=0.079 .
$$

Odderon. In the case of individual fits with the KN model, Table III and Fig. 5 show that with ensemble II the model predicts a crossing in $\sigma_{\text {tot }}(s)$, so that $\sigma_{\text {tot }}^{p p}$ becomes greater than $\sigma_{\text {tot }}^{\bar{p} p}$ above $\sqrt{s} \approx 50 \mathrm{GeV}: \Delta A=5 \pm 3$ and $\Delta B \sim 0$ (Sec. II C). However, as shown in Fig. 5, in this case the $\rho(s)$ data are not described ( $K=0$ or $K$ as a free parameter).

On the other hand, Table III shows that, in the case of global fits with both ensembles I and II, statistically, $\Delta A=0$ and $\Delta B=0$, so that $\Delta \sigma_{t o t}=\sigma_{t o t}^{\bar{p} p}-\sigma_{t o t}^{p p}=0$. However, from Figs. 6 and 7 , we see that, in both case, $K=0$ and $K$ as a free parameter, this model predicts a crossing in the $\rho(s)$ behavior, with $\rho_{p p}$ becoming greater than $\rho_{\bar{p} p}$ at $\sqrt{s} \approx 70-80 \mathrm{GeV}$ (for $K=0$ ) and $\sqrt{s} \approx 60-70 \mathrm{GeV}$ (for $K$ a free parameter), a result in agreement with the early fits by Gauron, Nicolescu, and Leader [45]. Most important, these results are also in complete agreement with all the experimental data presently available on $\sigma_{t o t}$ and $\rho$ and are independent of the ensemble used. This is certainly an interesting and important prediction that will be verified at the BNL Relativistic Heavy Ion Collider (RHIC) and the CERN Large Hadron Collider (LHC) [46].

Individual and global fits. From Tables II and III, with the exception of the individual fit to ensemble II with the $\mathrm{KN}$ model and $K$ as a free parameter, the statistical information does not indicate a preference between individual or global fits.

On the other hand, global fits clearly constrain the possible increase of the total cross section. For example, in the case of the DL model, with both ensembles I and II and $K=0$, we obtained

$$
\begin{gathered}
\epsilon_{\text {indi }}^{\mathrm{I}} \approx 0.088 \rightarrow \epsilon_{\text {simul }}^{\mathrm{I}} \approx 0.081 \quad(\text { reduction } \approx 9 \%) \\
\epsilon_{\text {indi }}^{\mathrm{II}} \approx 0.091 \quad \rightarrow \quad \epsilon_{\text {simul }}^{\mathrm{II}} \approx 0.083 \quad(\text { reduction } \approx 10 \%)
\end{gathered}
$$

As discussed previously, $\sigma_{\text {tot }}$ and $\rho$ do not have the same status as physical quantities, since the extracted $\rho$ value is model dependent. Therefore, in principle, we understand that global fits underestimate the possible rise of $\sigma_{t o t}$ in the asymptotic region. In our analysis the effects of the global and individual fits depend also on the subtraction constant, as discussed in what follows.

Subtraction constant. As commented before, if $K$ is taken as a free parameter, the fit procedure demands that it is correlated with all the other parameters of the model involved. Therefore, in principle, it is expected to have effects in both the low- and high-energy regions. Let us discuss our results through individual and global fits.

In the case of individual fits with the DL model, Fig. 2 shows that the results for $\rho(s)$ with $K=0$ and $K$ free are nearly the same above $\sqrt{s} \approx 40 \mathrm{GeV}$ and that below this energy the predictions are quite different. We shall not discuss the corresponding results with the KN model, since the data are not described (Fig. 5).

In the case of global fits with the DL model, beyond the differences in $\rho(s)$, here below $\sqrt{s} \approx 40 \mathrm{GeV}$, the asymptotic values of $\sigma_{t o t}(s)$ are different, namely, from Table II, 


$$
\begin{aligned}
& \left.\epsilon_{K=0}^{\mathrm{I}}=0.081 \pm 0.002 \quad \text { and } \quad \epsilon_{K \text { free }}^{\mathrm{I}}=0.083 \pm 0.02 \quad \text { (increase } \approx 2.5 \%\right), \\
& \left.\epsilon_{K=0}^{\mathrm{II}}=0.083 \pm 0.002 \quad \text { and } \quad \epsilon_{K \text { free }}^{\mathrm{II}}=0.084 \pm 0.02 \quad \text { (increase } \approx 1.2 \%\right) .
\end{aligned}
$$

In particular, with ensemble I, for $p p$ scattering at $\sqrt{s}=14 \mathrm{TeV}$ the fits indicate $\sigma_{t o t}^{p p} \sim 101 \mathrm{mb}$ for $K=0$ and $\sigma_{t o t}^{p p} \sim 104 \mathrm{mb}$ for $K$ as a free parameter. On the other hand, for global fits, the KN model is not so sensitive to the influence of the subtraction constant at least for $\rho(s)$ above $\sqrt{s} \approx 20 \mathrm{GeV}$, as shown in Figs. 6 and 7 . Therefore, in general, the subtraction constant affects the fit results in both the low- and high-energy regions. Since it is mathematically justified in order to control the convergence of the integral (or derivative) dispersion relation, we understand that the subtraction constant cannot be neglected in the analytical approach.

\section{Acknowledgments}

We are thankful to FAPESP for financial support (Contracts No. 00/13490-6, 00/00991-7, and No. 00/04422-7). We are also thankful to Professor M. M. Block for sending us the BHS results (Table I) and to Dr. J. Montanha Neto for a critical reading of the manuscript.

[1] Y. Dokshitzer, Philos. Trans. R. Soc. London A359, 309 (2001).

[2] G. Matthiae, Rep. Prog. Phys. 57, 743 (1994).

[3] R. J. Eden, Rev. Mod. Phys. 43, 15 (1971).

[4] S. M. Roy, Phys. Rep. 5, 125 (1972); J. Fischer, ibid. 76, 157 (1981); P. Valin, ibid. 203, 233 (1991).

[5] M. L. Goldberger, Y. Nambu, and R. Oehme, Ann. Phys. (N.Y.) 2, 226 (1957); P. Söding, Phys. Lett. 8, 285 (1964).

[6] U. Amaldi et al. Phys. Lett. 66B, 390 (1977).

[7] M. M. Block and R. N. Cahn, Rev. Mod. Phys. 57, 563 (1985).

[8] UA4/2 Collaboration, C. Augier et al. Phys. Lett. B 315, 503 (1993).

[9] M. Bertini, M. Giffon, L. Jenkovszky, and F. Paccanoni, Nuovo Cimento, Soc. Ital Fis., A 109, 257 (1996).

[10] N. V. Gribov and A. A. Migdal, Yad. Fiz. 8, 1002 (1968) [Sov. J. Nucl. Phys. 8, 583 (1969)]; J. B. Bronzan, in Argonne Symposium on the Pomeron, Report No. ANL/HEP-7327 (1973), p. 33; J. D. Jackson, in 1973 Scottish Summer School, Report No. LBL-2079, 1973, p. 39.

[11] J. B. Bronzan, G. L. Kane, and U. P. Sukhatme, Phys. Lett. 49B, 272 (1974).

[12] K. Kang and B. Nicolescu, Phys. Rev. D 11, 2461 (1975).

[13] K. Kang, P. Valin, and A. White, in Proceedings of the Vth Blois Workshop-International Conference on Elastic and Diffractive Scattering, Providence, 1993, edited by H.M. Fried, K. Kang, and C.-I. Tan (World Scientific, Singapore, 1994), p. 50; Nuovo Cimento Soc. Ital. Fis., A 107, 2103 (1994).

[14] J. R. Cudell, K. Kang, and S. K. Kim, Phys. Lett. B 395, 311 (1997); J. R. Cudell, V. V. Ezhela, K. Kang, S. B. Lugovsky, and N. P. Tkachenko, in Proceedings of the VIIIth Blois Workshop-International Conference on Elastic and Diffractive Scattering, Protvino, 1999, edited by V. A. Petrov and A. V. Prokudin (World Scientific, Singapore, 2000), p. 251; Phys. Rev. D 61, 034019 (2000); 63, 059901(E) (2001).

[15] J. R. Cudell et al., Phys. Rev. D 65, 074024 (2002); Phys. Rev. Lett. 89, 201801 (2002).

[16] R. F. Ávila, E. G. S. Luna, and M. J. Menon, Braz. J. Phys. 31, 567 (2001).

[17] R. F. Ávila, E. G. S. Luna, and M. J. Menon in LISHEP 2002-Workshop on Diffractive Physics, Rio de Janeiro, 2002, hep-ph/0206028 E. G. S. Luna and M. J. Menon, in New States of Matter in Hadronic Interactions, edited by H.-Thomas Elze et al. AIP Conf. Proc. 631, (AIP, Melville, NY, 2002) p.721; hep-ph/0105076

[18] A. Donnachie and P. V. Landshoff, Z. Phys. C 2, 55 (1979); Phys. Lett. B 387, 637 (1996).

[19] Particle Data Group, K. Hagiwara et al., Phys. Rev. D 66, 010001 (2002). The full data sets are available at http://pdg.lbl.gov

[20] T. K. Gaisser, Nucl. Phys. B (Proc. Suppl.) 12, 172 (1990).

[21] R. Engel, T. K. Gaisser, P. Lipari, and T. Stanev, Phys. Rev. D 58, 014019 (1998).

[22] T. K. Gaisser, U. P. Sukhatme, and G. B. Yodh, Phys. Rev. D 36, 1350 (1987).

[23] P. Sokolsky, Introduction to Ultrahigh Energy Cosmic Ray Physics Frontiers in Physics Vol 76 (Addison Wesley, New York, 1989).

[24] R. J. Glauber and G. Matthiae, Nucl. Phys. B21, 135 (1970).

[25] V. N. Gribov, Zh. Eksp. Teor. Fiz. 56, 869 (1969) [Sov. Phys. JETP 29, 483 (1969).

[26] R. M. Baltrusaitis et al., Phys. Rev. Lett. 52, 1380 (1984).

[27] H. Honda et al., Phys. Rev. Lett. 70, 525 (1993).

[28] J. Dias de Deus, Nucl. Phys. B59, 231 (1973); A. J. Buras and J. Dias de Deus, ibid. B71, 481 (1974).

[29] L. Durand and H. Pi, Phys. Rev. D 38, 78 (1988).

[30] L. Durand and H. Pi, Nucl. Phys. B (Proc. Suppl.) 12, 379 (1990). 
[31] T. T. Chou and C. N. Yang Phys. Lett. 128B, 457 (1983).

[32] N. N. Nikolaev, Phys. Rev. D 48, R1904 (1993).

[33] B. Z. Kopeliovich, N. N. Nikolaev, and I. K. Potashnikova, Phys. Rev. D 39, 769 (1989).

[34] M. M. Block, F. Halzen, and T. Stanev, Phys. Rev. Lett. 83, 4926 (1999); Phys. Rev. D 62, 077501 (2000).

[35] M. M. Block, R. Fletcher, F. Halzen, B. Margolis, and P. Valin, Phys. Rev. D 41, 978 (1990); M. M. Block, F. Halzen, and B. Margolis, Phys. Lett. B 252, 481 (1990); Phys. Rev. D 45, 839 (1992); M. M. Block, E. M. Gregores, F. Halzen, and G. Pancheri, ibid. 58, 017503 (1998); 60, 054024 (1999).

[36] C. L. Pryke, Astropart. Phys. 14, 319 (2001).

[37] M. M. Block (private communication).

[38] A. F. Martini and M. J. Menon, Phys. Rev. D 56, 4338 (1997).

[39] M. J. Menon, A. E. Motter, and B. M. Pimentel, Phys. Lett. B 451, 207 (1999).

[40] M. Froissart, Phys. Rev. 123, 1053 (1961).

[41] A. Martin, Nuovo Cimento A 42, 930 (1966); 44, 1219 (1966).

[42] G. Grunberg and T. N. Truong, Phys. Rev. Lett. 31, 63 (1973).

[43] L. Lukaszuk and B. Nicolescu, Lett. Nuovo Cimento Soc. Ital. Fis. 8, 405 (1973).

[44] D. Bernard, P. Gauron, and B. Nicolescu, Phys. Lett. B 199, 125 (1987).

[45] P. Gauron, B. Nicolescu, and E. Leader, Nucl. Phys. B299, 640 (1988).

[46] http://rhic.bnl.gov/pp2pp/ http://totem.web.cern.ch/Totem/ 